DEVELOPMENT OF A SCREENING METHOD FOR DROUGHT TOLERANCE IN COTTON SEEDLINGS

\author{
A Thesis \\ by \\ POLLY SUZANNE LONGENBERGER \\ Submitted to the Office of Graduate Studies of \\ Texas A\&M University \\ in partial fulfillment of the requirements for the degree of \\ MASTER OF SCIENCE
}

December 2005

Major Subject: Plant Breeding 


\title{
DEVELOPMENT OF A SCREENING METHOD FOR DROUGHT TOLERANCE IN COTTON SEEDLINGS
}

\author{
A Thesis \\ by \\ POLLY SUZANNE LONGENBERGER
}

\begin{abstract}
Submitted to the Office of Graduate Studies of
Texas A\&M University

in partial fulfillment of the requirements for the degree of
\end{abstract}

MASTER OF SCIENCE

$\begin{array}{ll}\begin{array}{l}\text { Approved by: } \\ \text { Co-Chairs of Committee, }\end{array} & \begin{array}{l}\text { C. Wayne Smith } \\ \text { Peggy Thaxton } \\ \text { Bobbie McMichael } \\ \text { Marla Binzel }\end{array} \\ \text { Head of Department, } & \text { C. Wayne Smith }\end{array}$

December 2005

Major Subject: Plant Breeding 


\begin{abstract}
Development of a Screening Method for Drought Tolerance in Cotton Seedlings.

(December 2005)

Polly Suzanne Longenberger, B.S., The Pennsylvania State University

Co-Chairs of Advisory Committee: Dr. C. Wayne Smith

Dr. Peggy Thaxton
\end{abstract}

The key to an efficient screening method is the ability to screen large amounts of plant material in the shortest time possible. Unfortunately, due to the complexity of drought tolerance, a quick and effective screen for this trait has yet to be established. The research reported herein was designed to evaluate a screening method for drought tolerance in cotton (Gossypium hirsutum L.) seedlings. Twenty-one converted race stocks (CRS) and two cultivars were evaluated for seedling drought tolerance on an individual plant basis. CRS are day-sensitive primitive lines derived from various wild race stocks that were converted to day neutrality for use in temperate region plant improvement programs (McCarty et al., 1993). Genotypes were evaluated October November 2004 and February - March 2005 under greenhouse conditions at the Norman E. Borlaug Center for Southern Crop Improvement, College Station, TX. Seedlings were subjected to three sequential cycles of drought at 15 days after planting (DAP). Drought cycles consisted of withholding water until the moisture content of "indicator" cone-tainers, containing Deltapine 491 (DP 491), had an average volumetric water content of 0.07. Plants were then watered to field capacity and percent survival was recorded after 48 hours. Genotypes differed in their percent survival following three 
consecutive drought cycles. Drought cycles 2 and 3 did not contribute to the separation of genotypes. DP 491 was the most tolerant genotype evaluated. None of the CRS were more or less tolerant than Acala 1517-99. CRS M-9044-0165 was the most stable genotype across the two experiments. 
To Carl 


\section{ACKNOWLEDGEMENTS}

I would like to thank Dr. C. Wayne Smith for sharing his expertise and for guiding my research. He is a gifted advisor and I am pleased to have had the opportunity to study in his program. Dr. Peggy Thaxton has been a valuable mentor and role model. She was always available for consultation on issues related to my project.

Sincere thanks are extended to Dr. Bobbie McMichael for his advice on the seedling drought protocol. I would also like to thank Dr. Marla Binzel for her participation in this research.

I would like to thank the staff of the Cotton Improvement Lab and all student workers for their help in completing this project. My fellow graduate students, Chris Braden, Brian Schwartz, and Chris Souder, also deserve my thanks for their help and advice.

Lastly, I would like to thank Dr. Sara Duke for her guidance and expertise. She was generous to give her time to help me with my statistical analyses. 


\section{TABLE OF CONTENTS}

ABSTRACT $\ldots \ldots \ldots \ldots \ldots$




\section{LIST OF FIGURES}

FIGURE

Page

1 Percent survival of groups of fourteen Deltapine 491 cone-tainers rewatered at specific volumetric water contents of twenty-eight indicator Deltapine 491 cone-tainers under growth chamber conditions at College Station, TX in 2004.

2 Mean number of surviving plants of twenty-one CRS and two cotton cultivars following three consecutive drought cycles versus percent survival

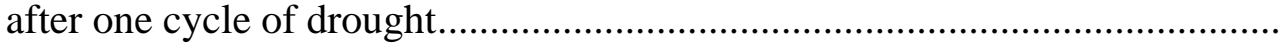




\section{LIST OF TABLES}

TABLE

1 Accession number, PI number, race designation, and origin of cotton accessions screened for seedling drought tolerance

2 Variance analysis for percent survival of twenty-one converted race stocks and two cotton cultivars after three cycles of drought in two experiments conducted under greenhouse culture at College Station, TX, in 2004 and 2005

3 Percent survival twenty-one converted race stocks and two cotton cultivars after one cycle of drought in two experiments conducted under greenhouse culture at College Station, TX, in 2004 and 2005.

4 Difference in percent survival of twenty-one converted race stocks and two cotton cultivars after one cycle of drought in two experiments conducted under greenhouse culture at College Station, TX, in 2004 and 2005............... 


\section{INTRODUCTION}

Effective screening methods must evaluate plant performance at critical developmental stages, be completed rapidly, use small amounts of plant material, and screen large numbers of plants (Johnson, 1980). And perhaps most importantly, the screening method must be incorporated into plant breeding programs to facilitate meaningful genetic improvement. Researchers are still looking for a screening method for drought tolerance in crop plants that fulfills all of these important requirements.

Water is the most important factor limiting crop productivity throughout the world. The demand for drought tolerant genotypes will be exacerbated as water resources and the funds to access them become limited. The burden falls upon crop physiologists to understand detrimental effects of drought on plant processes and to convey their findings to plant breeders for the development of tolerant genotypes. Difficulties in the past have included the identification of physiological characteristics that are correlated with drought stress and thus as such are indicators of drought tolerance. Physiologists are able to measure various plant characteristics that correlate with drought tolerance, such as water use efficiency, root structure, detached leaf water loss, leaf water potential, stomatal characteristics, and osmotic adjustment, but the tests to determine these characteristics are too tedious or time consuming for a plant breeder to evaluate segregating populations. There is a need for a protocol to determine the drought tolerance of cotton germplasm. In this study, cotton seedlings were evaluated on an individual plant basis to verify their tolerance to periods of water deficit.

This thesis follows the style and format of Crop Science. 


\section{Research Objectives}

The objectives of this study were to [1] develop a protocol to evaluate cotton seedlings for drought tolerance on an individual plant basis and [2] compare a number of CRS of upland cotton with two cultivars representing diverse germplasm pools for seedling drought tolerance. 


\section{LITERATURE CITED}

Crop plants commonly are subjected to periodic water deficits. Since society is reliant on these plants for food and feed, their ability to survive and produce during periods of water deficit is of interest to scientists as well as society in general. For this reason, many researchers have focused on the improvement of drought tolerance in crop species. Measuring drought tolerance under field conditions is difficult due to variation in weather conditions throughout the growing season and from year to year. Between and within field soil type variation also complicates field drought tolerance evaluation. This has led to efforts of drought simulation in greenhouses and growth chambers.

A growth chamber programmed to simulate the first 10 days in August in the arid Southwest was used to select for seedling drought tolerance among three species from two genera of range grasses (Wright, 1964). Wright's growth chamber selection technique separated the species into the same order as their natural range performance. The ability to select among large numbers of seedlings and the capacity to control environmental conditions were cited by the author as the most important benefits of his protocol. Wright and Jordan (1970) used Wright's 1964 method to select among boer lovegrass (Eragrostis curvula Nees) seedlings for drought tolerance. The most tolerant selection of 16 clones evaluated was assessed for range performance and was found to be superior to the check cultivar.

Wright and Brauen (1971) attempted to identify associations between seedling drought tolerance, as determined by the programmed chamber method of Wright (1964), and plant characteristics. Thirty-six lovegrass lines and a commercial cultivar, 
Lehmann, were evaluated using the chamber method, and surviving plants were compared for plant characteristics such as growth habit, foliage color, anther color, chromosome number, seed weight, and seed dormancy. None of the characteristics studied provided adequate association with seedling drought tolerance that would validate its use as a selection criterion.

The drought tolerance of forage grasses was appraised in a growth chamber by watering to field capacity and then withholding water until the soil-filled trays containing the plants reached a predetermined weight (Tischler et al., 1991). Although this method gave consistent results, the authors expressed concern that the tray system did not allow for the extraction of water by long, deep roots in a deep soil profile and that additional techniques were needed to assess such drought-avoidance mechanisms.

Lichthardt and Weaver (1985) assessed range grass seedlings with varying leaf numbers (varying age) for drought tolerance by monitoring their survival after a two week long drought. The authors found significant differences among the four genotypes tested when plants with more than four leaves were tested. The authors stress that measuring drought tolerance in pots as they did does not test all aspects of drought tolerance since plants have limited soil to explore for water.

Eight corn lines were evaluated under controlled heat conditions in an environmental chamber (Hunter et al., 1936). Injury ratings and subsequent rankings of the lines were similar for both the controlled heat experiments and previous field observations. The authors state that the development of a simple seeding test to determine drought tolerance would be a valuable tool for corn breeders who consider 
tolerance to hot and dry conditions a top priority. A similar study was performed and expanded into a genetic study of the inheritance of heat tolerance. Corn strains with known field-based drought classifications and $F_{1}$ progeny from crosses among these strains were tested for their response to controlled high temperature treatments to confirm the drought tolerance ratings found during field studies (Heyne and Brunson, 1940). Observations of plant injury, survival, and recovery were made. Essentially the same order of relative resistance among the strains was found in the controlled heat experiments as in mature plants subjected to drought and heat in the field. The authors summarize that heat tolerance was heritable and that in most cases it was controlled by dominant gene action.

A high heat and low humidity treatment in a forced air dryer was used to test seedlings of eight sweet and four dent corn lines that had varying levels of field drought tolerance (Kilen and Andrew, 1969). Significant correlations were found between heat test injury ratings and field drought classifications.

Sammons et al. (1978) screened twenty soybean cultivars believed to vary in their drought tolerance. The experiment was conducted in a growth chamber and pots were premoistened to three different soil water potentials, $-0.07 \mathrm{MPa}$ (control), $-0.3 \mathrm{MPa}$ (intermediate moisture stress), and -0.89 MPa (severe moisture stress). Water potential, leaf area and dry weight, and photosynthesis were measured. Significant cultivar x moisture treatment interactions were found for all plant characteristics, and cultivars were not consistently categorized by the variables measured. The same authors later screened the same twenty soybean cultivars in a large wooden box $(1.5 \times 3.7 \times 0.6 \mathrm{~m})$ 
under greenhouse conditions (Sammons et al., 1979). Water was withheld for the duration of the experiment (30 days). Leaf lamina expansion rate and plant growth rate were measured, neither of which consistently classified the cultivars relative to drought tolerance. The authors concluded that a combination of characteristics must be evaluated to assess drought tolerance.

Researchers often utilize boxes in which to establish plants for drought evaluation. Boxes $(1.5 \times 3.7 \times 0.6 \mathrm{~m})$ were used to differentiate between seven alfalfa cultivars and three germplasm sources for forage yield and root characteristics under three moisture treatments in a shadehouse (Salter et al., 1984). Forage yield decreased by 30 and $50 \%$ in the intermediate and low moisture treatments, respectively, compared to the high moisture treatment. There were no differences between genotypes in the low moisture treatment. Root fibrousness increased and root weight decreased with increased moisture stress. All root characteristics correlated with forage yield and the authors determined that the added time and expense of root excavation was not worth the additional information.

Fifteen-day-old cotton seedlings were subjected to four, 4-day drought cycles in a growth chamber to determine drought tolerance (Penna et al., 1998). The authors used soil filled trays $(50 \times 35 \times 9 \mathrm{~cm})$ containing 12 rows of plants with 12 plants per row. Significant differences in seedling survival were found but results were not consistent across repeated experiments. An unfortunate aspect of using boxes for drought evaluation is that plants compete for limited soil moisture. Evaluating individual plants 
or planting single genotypes per box will avoid the issue of root performance or size differences among the genotypes tested.

Physiological parameters have been tested in an effort to determine drought tolerance. Root growth and excised leaf water loss of upland cotton seedlings were evaluated under non-stressed and stressed conditions in a greenhouse by Basal et al. 2005. Plants were grown in individual tubes, $11 \mathrm{~cm}$ diameter $\times 70 \mathrm{~cm}$. Stress conditions involved two consecutive drought cycles in which the plants were watered to field capacity 12 hours after visual signs of wilting. The authors found differences among genotypes for root growth and excised leaf water loss, and recommend the use of a line exhibiting a robust root system and the ability to maintain high excised leaf water content as a parent in developing cultivars adapted to dry environments. Roark et al. (1975) reported that cultivars developed in semiarid regions had faster rates of water loss during the stomatal phase of transpiration, reached a lower relative water content before stomatal closure, and lost more water before the stomates closed than did detached leaves of cultivars developed in irrigated or high rainfall regions. A negative correlation was found between relative water content at mean stomatal closure and growth rate under water stressed field conditions, indicating that plants closing their stomata at lower relative water content values may produce higher levels of dry matter (Quisenberry et al., 1982).

Oosterhuis and Wullschleger (1987) examined the magnitude and duration of osmotic adjustment in cotton leaves and roots in response to water stress during and after the stress application. Osmotic adjustment is linked to the maintenance of turgor in crop 
plants experiencing water deficit. The authors found that osmotic adjustment occurred in leaves and in roots of cotton plants that were subjected to water deficit under growth chamber conditions. Leaf osmotic potential was found to return to normal levels more rapidly than root osmotic potential during recovery from water stress. Turgor maintenance may allow for continued growth during water shortages. The experiment was performed on only a single genotype.

Exotic cottons were evaluated in the field under irrigated and non-irrigated conditions for shoot dry weight, in the laboratory for heat tolerance, and in the greenhouse for root growth (Quisenberry et al., 1981). Significant variability occurred among the strains tested for all measurements. The authors suggested that root morphology and root growth potentials appear important for the adaptation of cotton to water-limited environments. This belief is further supported by Pace et al. (1999) who found drought-stressed cotton plants had longer but narrower taproots than non-stressed plants. The authors suggested that this may allow plants to access water deep within the soil profile.

Cook and El-Zik (1992) examined root growth of six cotton genotypes under field conditions at the seedling stage and first bloom stage of growth to determine the relationship between root growth and drought-influenced boll abscission and lint yield. Root system parameters differed among genotypes at both growth stages. A positive association was found between drought-induced boll abscission and root-to-shoot ratio at first bloom and a negative association was found between dryland lint yield and first bloom root-to-shoot ratio. The authors conclude that genotypes with rapid root 
establishment prior to fruiting stage would enhance their ability to export photosynthates to developing bolls.

Leaf turgidity was studied as it relates to drought tolerance in cotton by measuring biomass production and yield in field and greenhouse studies by Quisenberry et al. (1985). Two photoperiodic cotton strains were identified as having varying leaf turgidity under water deficient conditions. It was found that the non-wilting strain had higher biomass production and yield than the wilting strain under drought conditions.

Kaul (1966) studied the relationship between water stress and respiration in wheat. He measured the respiration of one drought tolerant and one drought susceptible cultivar under stressed conditions and found that both cultivars had similar respiration responses. The same author later (1969) performed field experiments examining the relationship between water potential, osmotic pressure, and yield for six wheat cultivars, reporting no consistent positive or negative relationship between plant water status and yield.

Polyethylene glycol (PEG) often is used in laboratories to simulate water deficit. PEG enables researchers to grow plants in solutions of known water potentials. The use of PEG solutions as growth media especially is useful in drought research concerned with root growth and structure since the roots are more easily accessed for analysis when plants are grown in PEG solution rather than in soil. Four cotton genotypes with varying levels of suspected drought tolerance were subjected to a water deficit of $-0.3 \mathrm{MPa}$ induced by PEG (Nepomuceno et al., 1998). Water potentials and osmotic potentials of leaves and roots of stressed and non-stressed controls were measured. The tolerant 
genotypes maintained higher water potentials during drought stress, allowing them to maintain photosynthesis, stomatal conductance, and relative water content at near unstressed levels. The authors believe that their study supports the use of physiological parameters as a means to select and improve germplasm.

Germination of sorghum in D-Mannitol, sugar, and salt solutions was tested as an indicator of drought hardiness (Vasudevan and Balasubramaniam, 1964). Sorghum strains developed under rain fed conditions had higher germination percentages than those strains developed under irrigated conditions.

Although much effort has been put forth to develop methods for identifying drought tolerant genotypes, cotton breeders are still in need of a quick, easy, and accurate screen for drought tolerance. The problem becomes how to determine what, how, when, and where to measure the plant characteristics that will correlate with high productivity under water deficiency conditions. 


\section{MATERIALS AND METHODS}

Twenty-one day-neutral converted cotton accessions were tested for seedling drought tolerance (Table 1) (Liu et al., 2000). The conversion of 79 primitive race stock accessions was reported by McCarty and Jenkins (1993). The photoperiodic primitive race stocks were crossed with Deltapine 16 and day-neutral plants were selected in the $\mathrm{F}_{2}$. Day-neutral progenies were then backcrossed four times to the primitive parent. Selection for day-neutrality occurred in the $F_{2}$ following each backcross. Each CRS has a designation beginning with the letter $\mathrm{M}$, followed by four digits which indicate the year of release and the $\mathrm{BC}_{n} \mathrm{~F}_{n}$ generation at the time of release. The last four digits correspond to the Texas (T-) Cotton Germplasm Collection accession number of the unconverted primitive stock (Basal et al., 2003). The CRS seed source was increase plots planted in Weslaco, TX in 2003.

All CRS, with the exception of M-8844-0096, were found to be the ten most tolerant and ten least tolerant in a survey of 79 CRS for seedling drought tolerance using an alternate protocol (unpublished data). M-8844-0096 was found to be heat tolerant in a previous field experiment testing seed set efficiency during the extremely hot growing season of 1998 in College Station, TX (unpublished data).

Two cultivars, Acala 1517-99 and DP 491, were included to represent two distinct germplasm pools. Acala 1517-99 was released by the New Mexico Agricultural Experiment Station in 1999. It was developed to produce high yield and superior fiber quality in New Mexico under conventional irrigated culture. The origin of Acala 1517- 
Table 1. Accession number, PI number, race designation, and origin of cotton accessions screened for seedling drought tolerance.

\begin{tabular}{llllll}
\hline $\begin{array}{l}\text { Day-neutral } \\
\text { Accession } \\
\text { number }\end{array}$ & PI no. & \multicolumn{1}{c}{ Race } & \multicolumn{3}{c}{ Origin } \\
\hline M-9044-0007 & PI561950 & Latifolium & Mexico & NC† & NC \\
M-9044-0017 & PI561951 & Latifolium & Mexico & Chiapas & NC \\
M-9044-0024 & PI561952 & Latifolium & Mexico & Chiapas & NC \\
M-9044-0031 & PI561954 & Latifolium & Mexico & Chiapas & Acala \\
M-9044-0033 & PI561956 & Latifolium & Mexico & Chiapas & Acala \\
M-8844-0055 & PI561963 & Latifolium & Mexico & Chiapas & Comitan de Dominguez \\
M-9044-0057 & PI561964 & Latifolium & Mexico & Chiapas & San Bartalome \\
M-9044-0060 & PI561965 & Latifolium & Mexico & Chiapas & San Bartalome \\
M-9044-0061 & PI561966 & Latifolium & Mexico & Chiapas & San Bartalome \\
M-9044-0072 & PI561971 & Latifolium & Guatemala & Suchitepequez & San Jose el Idolo \\
M-8844-0096 & PI561979 & Latifolium & Guatemala & Jutiapa & Santa Catarina Mita \\
M-9044-0140 & PI561991 & Latifolium & Guatemala & Jutiapa & Jutiapa \\
M-9044-0150 & PI561992 & NC $\dagger$ & Guatemala & Jutiapa & Progreso \\
M-9044-0165 & PI562000 & Punctatum & Guatemala & Zacapa & Las Carretas \\
M-8744-0175 & PI562004 & Latifolium & Guatemala & Jutiapa & Santa Catarina Mita \\
M-9044-0180 & PI562005 & Latifolium & Guatemala & Santa Rosa & NC \\
M-9044-0206 & PI562008 & Latifolium & Mexico & Guerrero & Maquina del Rio de Niepa \\
M-8844-0243 & PI562015 & Latifolium & Mexico & Oaxaca & NC \\
M-9044-0244 & PI562016 & Latifolium & Mexico & Oaxaca & Ixcopa \\
M-8744-0257 & PI562019 & Morrilli & Mexico & Oaxaca & Mitla \\
M-9044-0570 & PI562021 & NC & Sudan & NC & NC \\
\hline$\dagger$ NC den0tes & & & & \\
\hline
\end{tabular}

$\dagger$ NC denotes information not contained in collection. 
99 was from a single plant selection from experimental B2541 by C.L. Roberts. The cross B742/E1141 gave rise to B2541. B742 was derived from Acala 9136/250, with Acala 9136 reportedly having significant introgression from Gossypium barbadense L. cv. Tanguis. E1141 and 250 are of unknown origin (Cantrell et al., 2000). DP 491 was released by Delta and Pine Land Company in 2002. It is marketed as a picker cultivar with high gin turnout and low micronaire suitable for all states in the Cotton Belt with the exception of Arizona.

A preliminary experiment was performed to determine the volumetric water content required to cause 50 \% recovery of DP 491. 168 Ray Leach “Cone-tainers”TM (3.8 cm diameter $\times 14 \mathrm{~cm}$ depth) were filled by volume with 83 cc of fritted clay (Absorb-N-Dry, Flatonia, TX). The experiment was performed in an environmental growth chamber with a 12 -hr day length, $32^{\circ} \mathrm{C}$ day $/ 25^{\circ} \mathrm{C}$ night and $80 \%$ relative humidity at the Norman E. Borlaug Center for Southern Crop Improvement, College Station, TX. Two DP 491 seeds were sown per cone-tainer and thinned to one plant per cone-tainer after germination. Cone-tainer trays (96 cone-tainers per tray) were rotated daily to minimize variation due to microclimates in the growth chamber. All Conetainers were watered to field capacity daily until 15 DAP. Twenty-eight of the Conetainers were weighed periodically to monitor water loss. At approximately $120 \mathrm{~h}$ post field capacity a group of 14 Cone-tainers were rewatered and percent recovery was recorded after 48 hours. This process was repeated until all remaining cone-tainers had been rewatered with the last group being rewatered at $245 \mathrm{~h}$ post field capacity. The plot of percent survival versus volumetric water content generated from this experiment 
indicated that an average volumetric water content of 0.08 was required for $50 \%$ recovery of DP 491 (Figure 1).

A preliminary replication of the drought screen experiment was performed under greenhouse conditions following the procedure outlined in the following paragraph. The indicator DP 491 Cone-tainers were allowed to reach an average volumetric water content of 0.08. This did not cause any mortality among the DP 491 plants in the test and only limited mortality among the CRS and Acala 1517-99 (data not shown). Therefore, the average volumetric water content was lowered to 0.07 for the experiments described in this thesis.

Two experiments were performed. Experiment 1 was conducted October November 2004 and experiment 2 was conducted February - March 2005. Experiments 1 and 2 were performed under greenhouse conditions with $31^{\circ} \mathrm{C} / 24^{\circ} \mathrm{C}$ and $31^{\circ} \mathrm{C} / 23^{\circ} \mathrm{C}$ (day/night), respectively, at the Norman E. Borlaug Center for Southern Crop Improvement, College Station, TX. Cotton seedlings were evaluated for drought tolerance on an individual plant basis. Ray Leach “Cone-tainers”TM (3.8 cm diameter x $14 \mathrm{~cm}$ depth) were filled by volume with 83 cc of fritted clay (Absorb-N-Dry, Flatonia, TX). Two seeds were sown per cone-tainer and thinned to one plant per cone-tainer after germination. Four replications were evaluated during each experiment. Each replication consisted of 14 plants of each converted race stock and Acala 1517-99. Each replication included 56 plants of DP 491 so that a better estimate of average volumetric water content could be obtained. 


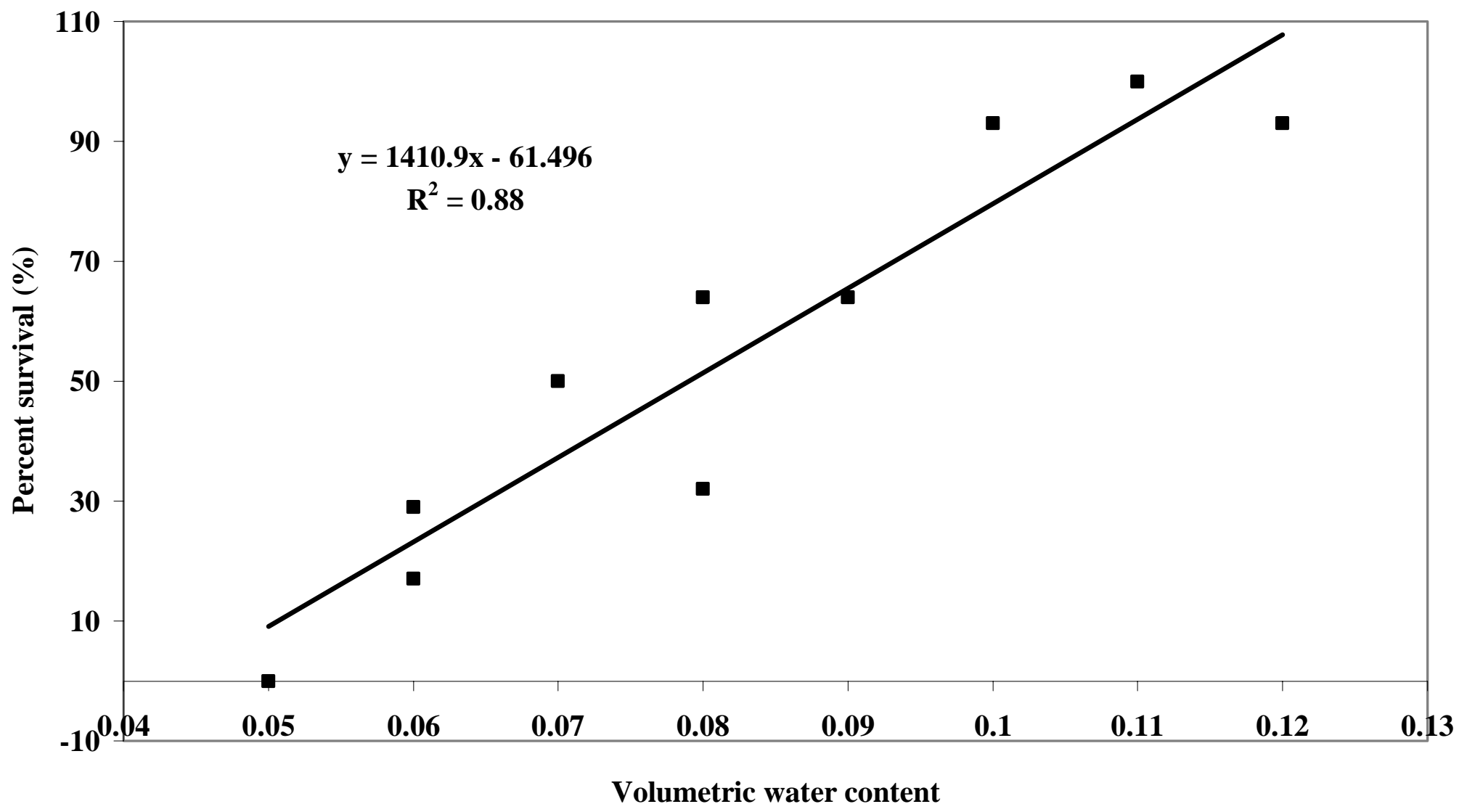

Figure 1. Percent survival of groups of fourteen Deltapine 491 cone-tainers rewatered at specific volumetric water contents of twenty-eight indicator Deltapine 491 cone-tainers under growth chamber conditions at College Station, TX in 2004. 
Trays holding the Cone-tainers were rotated daily to minimize variation caused by microclimates in the greenhouse. All Cone-tainers were watered to field capacity daily until 15 DAP when the seedlings were subjected to three sequential cycles of drought. Drought cycles consisted of withholding water until the moisture content of the 56 “indicator" Cone-tainers, containing DP 491, had an average volumetric water content of 0.07. Plants were then watered to field capacity and percent survival was recorded after 48 hours. All plants, including the “indicator” DP 491 plants were subject to stress or death due to the drought treatment. DP 491 plants that did not survive a drought cycle did not contribute to the volumetric water content estimate in future cycles. Surviving plants were defined as having least one turgid leaf and a live apical meristem. Percent survival following each drought cycle was determined by taking the number of survivors following the drought divided by the number of plants that were alive at the start of the drought cycle.

Since the response variable was binomial in nature, i.e. each plant was alive or dead after each drought cycle, the data were analyzed with mixed-effects logistic regression in SAS using the GLIMMIX procedure (release 9.1.3) (SAS Institute, 2004). The analysis included experiment, genotype, and drought cycle as fixed effects and replication nested in experiment as a random effect. 


\section{RESULTS AND DISCUSSION}

The ANOVA indicated that percent survival differed among the twenty-three genotypes (Table 2). This combined analysis for three drought cycles and two experiments showed a difference among drought cycles. The average percent survival for drought cycles 1, 2, and 3 across both experiments were 47, 94, and 89, respectively. The largest amount of plant mortality occurred after the first drought cycle in each experiment. Individualized analysis of droughts showed that differences among genotypes were found in drought one but no differences were found among genotypes for droughts 2 or 3 . This indicates that no further information was gained from drought cycles 2 and 3. A plot of the mean number of survivors after drought cycle 3 versus percent survival after drought cycle 1 shows a linear correlation (Figure 2). Therefore, the genotypes with high percent survival after drought cycle one will also have a high number of final survivors after drought cycle 3. Plant breeders would be interesting in making selections from among these final survivors.

To avoid inflation of the survival data by including cycles 2 and 3 only the means from drought cycle one are separated at $\mathrm{P}<0.05$ (Table 3). DP 491 was the most tolerant genotype with 82 percent survival. Acala 1517-99 had a percent survival of 50 . None of the CRS were more tolerant or susceptible than Acala 1517-99. M-8744-0175 was the most tolerant CRS with a percent survival of 60 but was not different than 16 of the other CRS and Acala 1517-99.

A Fisher's LSD was performed to determine the significance of experiment $\times$ genotype interaction (Table 4). Since the generalized linear mixed model output does 
Table 2. Variance analysis for percent survival of twentyone converted race stocks and two cotton cultivars after three cycles of drought in two experiments conducted under greenhouse culture at College Station, TX, in 2004 and 2005.

\begin{tabular}{llc}
\hline Source & \multicolumn{1}{c}{ df } & \multicolumn{2}{c}{ Percent survival } \\
\hline & & $\boldsymbol{F}$ \\
Cycle & 2,563 & $428.26^{* * *}$ \\
Genotype & 22,563 & $13.82^{* * *}$ \\
Experiment & 1,6 & 1.65 \\
Experiment $\times$ Genotype & 22,563 & $2.96^{* * *}$ \\
\hline *** Significant at the 0.0001 & probability level.
\end{tabular}




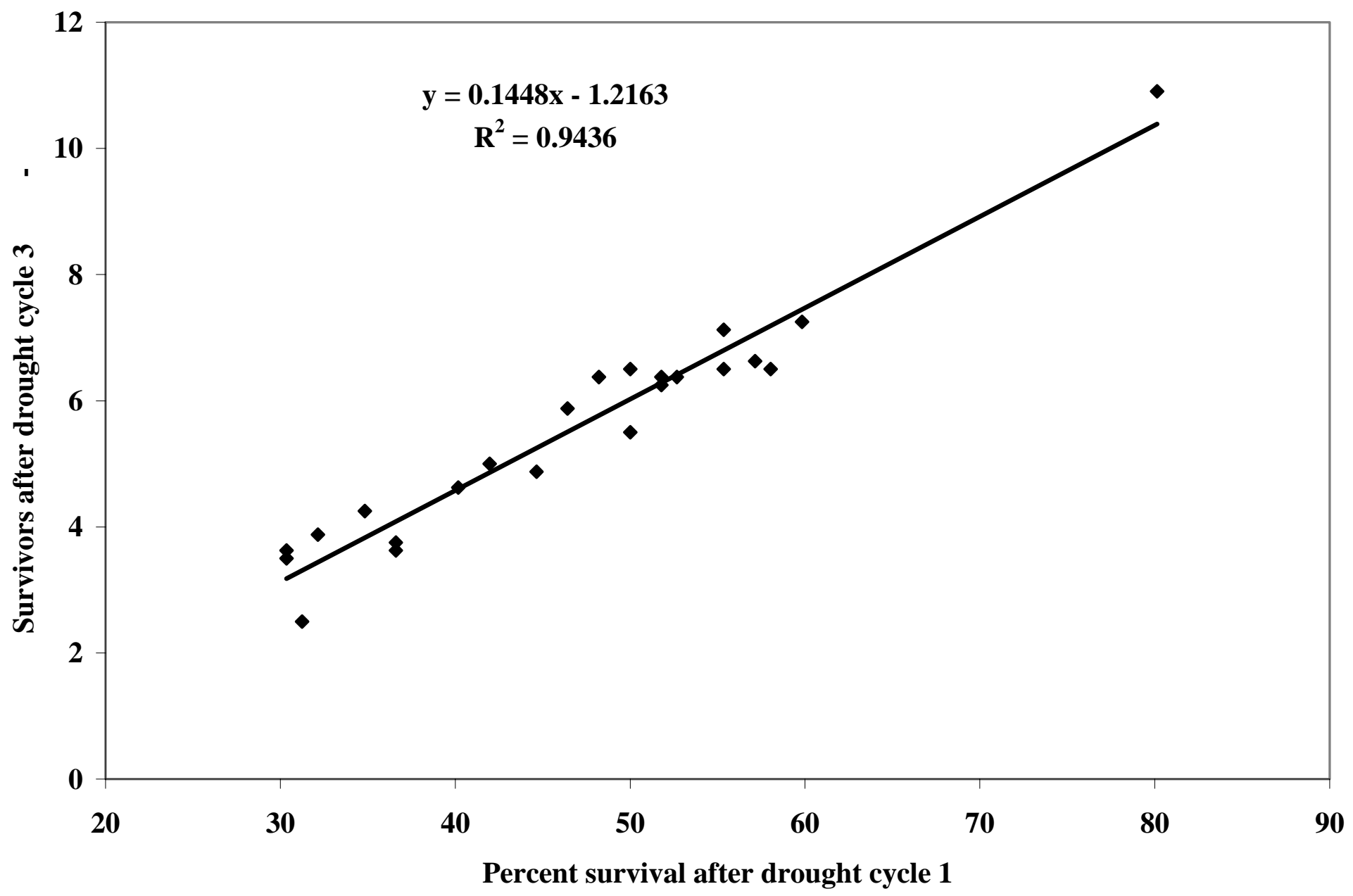

Figure 2. Mean number of surviving plants of twenty-one CRS and two cotton cultivars following three consecutive drought cycles versus percent survival after one cycle of drought. 
Table 3. Percent survival of twenty-one converted race stocks and two cotton cultivars after one cycle of drought in two experiments conducted under greenhouse culture at College Station, TX, in 2004 and 2005.

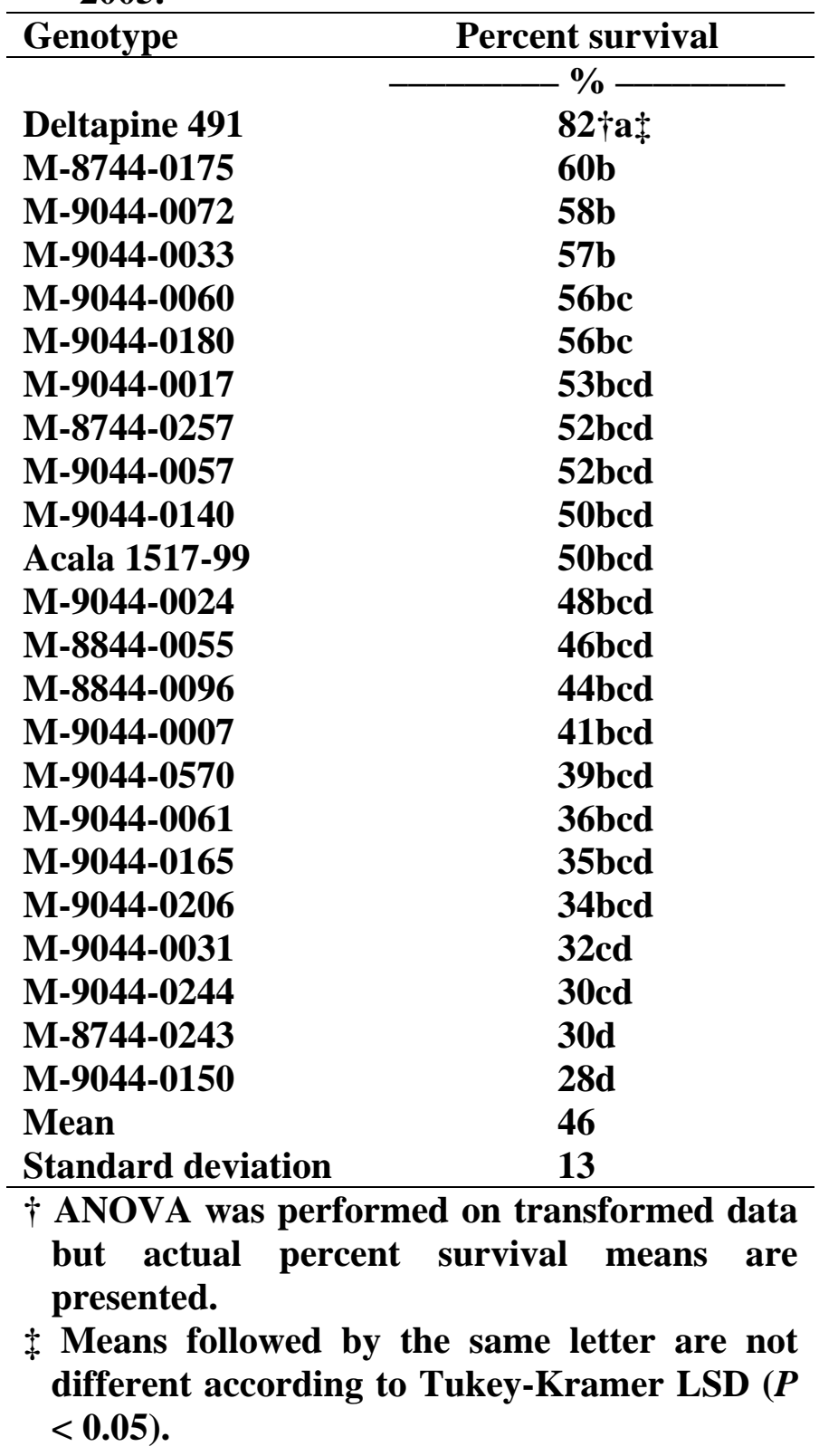


Table 4. Difference in percent survival of twenty-one converted race stocks and two cotton cultivars after one cycle of drought in two experiments conducted under greenhouse culture at College Station, TX, in 2004 and 2005.

\begin{tabular}{|c|c|c|c|}
\hline \multirow[t]{2}{*}{ Genotype } & \multicolumn{2}{|c|}{ Percent survival } & \multirow{2}{*}{ difference } \\
\hline & $\overline{0}$ & & \\
\hline M-9044-0206 & $54 \dagger$ & 19 & $35 a \neq$ \\
\hline M-9044-0060 & 70 & 41 & 29ab \\
\hline M-9044-0150 & 45 & 16 & 29ab \\
\hline M-9044-0140 & 63 & 37 & 26abc \\
\hline M-9044-0570 & 52 & 28 & 24abc \\
\hline M-9044-0180 & 65 & 46 & 19abcd \\
\hline M-8844-0055 & 55 & 37 & 18abcd \\
\hline M-9044-0244 & 39 & 23 & 16abcd \\
\hline Deltapine 491 & 88 & 73 & 15abcd \\
\hline M-8844-0096 & 52 & 37 & 15abcd \\
\hline M-9044-0072 & 65 & 52 & 13abcde \\
\hline M-8744-0175 & 66 & 54 & 12abcde \\
\hline M-9044-0024 & 54 & 43 & 11abcde \\
\hline M-8744-0243 & 35 & 25 & 10abcde \\
\hline M-9044-0057 & 54 & 50 & 4bcdef \\
\hline M-9044-0165 & 35 & 34 & 1bcdef \\
\hline M-8744-0257 & 50 & 54 & $-4 c d e f$ \\
\hline M-9044-0031 & 28 & 35 & $-7 \mathrm{def}$ \\
\hline M-9044-0033 & 54 & 61 & $-7 \mathrm{def}$ \\
\hline M-9044-0061 & 28 & 45 & $-17 \mathrm{ef}$ \\
\hline Acala 1517-99 & 39 & 61 & $-22 f$ \\
\hline M-9044-0007 & 30 & 54 & $-24 f$ \\
\hline M-9044-0017 & 39 & 66 & $-27 f$ \\
\hline Mean & 50 & 43 & \\
\hline Standard deviation & 15 & 15 & \\
\hline Range & 88-27 & 73-16 & \\
\hline
\end{tabular}

$\dagger$ LSD was performed on transformed data but actual percent survival means are presented.

$¥$ Means followed by the same letter are not different according to Fisher's LSD $(P<0.05)$. 
not supply the mean square error and degrees of freedom from the experiment $x$ genotype interaction, a general linear model (GLM) analysis was performed on the logit transformations of the survival data collected after one drought cycle. The logit transformation was selected to normalize the data since it is also the transformation performed in the GLIMMIX procedure. The logit transformation is used in logistic regression since it has many of the desirable properties of a linear regression model. It is linear in its parameters, may be continuous, and may range from $-\infty$ to $+\infty$, depending on the range of the response variable (Hosmer and Lemeshow, 1989).

The test indicated that percent survival of some genotypes were differential across the two experiments. There was not a uniform response among genotypes in direction or magnitude across experiments. Differences in direction are illustrated by seven of the genotypes having a negative difference in percent survival across experiments indicating that their percent survival was lower in experiment two than it was in experiment one. The remaining 16 genotypes had a higher percent survival in experiment two than in experiment one.

CRS M-9044-0165 was the most stable genotype across experiments with a difference in percent survival between experiments of 1 . No genotypes had a significantly higher difference in percent survival than M-9044-0165 and only one genotype, M-9044-0206, had a significantly higher difference at 35. DP 491, with a difference of 15, was more stable across the two experiments than Acala 1517-99 which had a difference of -22. Fourteen of the CRS were also more stable than Acala 1517-99. 
None of the CRS showed more stability than DP 491. M-9044-0061, M-9044-0007, and M-9044-0017 showed less stability than DP 491.

Visual observations during both experiments indicated that the CRS and Acala 1517-99 grew at a slightly faster rate than DP 491. DP 491 plants seemed to maintain their first true leaf and their cotyledons during the drought cycles while the CRS and Acala 1517-99 continued to elongate and put on two true leaves. This continued development during drought may have led to the lower survival percentages observed among the CRS and Acala 1517-99. Though DP 491 seedlings were able to survive the drought cycles, it appears they did so at the cost of growth and development. Both types of drought tolerance exhibited by the genotypes can be adventitious depending on the specific situation. For instance, the tolerance exhibited by DP 491 would be beneficial if one severe drought were to be experienced and the plants had time during the remainder of the season to make up for lost growth and development. However, if numerous short drought periods were to occur genotypes like the CRS and Acala 1517-99 would be preferred since they will continue to grow during the drought periods and are kept alive by intermittent rain or irrigation events. 


\section{CONCLUSIONS}

A protocol was developed to screen cotton seedlings for drought tolerance on an individual plant basis. The rankings for separate trials of the protocol were similar but not completely in agreement. Evaluation of genotypes under growth chamber conditions may allow for better control over temperature and humidity. A more controlled environment may reduce or eliminate the genotype $\times$ experiment interaction. Also, two out of the three drought cycles were longer in duration (hours post field capacity) in experiment one than in experiment two. These differences may have added to the genotype $\times$ experiment interaction. Though the plants were rewatered when the indicator DP 491 Cone-tainers reached 0.07 volumetric water content for each drought cycle, the difference in the length of the cycle may have triggered different physiological responses among the genotypes. Further trials of the protocol and additional evaluation of the genotypes by other methods may further validate the credibility of this method as a dependable screening tool.

It was determined that drought cycles two and three did not provide further information relative to the seedling drought tolerance of the genotypes in the test. Therefore, one drought cycle may be sufficient to separate cotton genotypes for seedling

drought tolerance. A second drought cycle (whose duration would be at the discretion of the researcher) after the initial screening could allow for the selection of only the most tolerant individuals of each genotype for advancement to future screens. Alternatively, selections could be made among the survivors of a single drought cycle and their offspring could be subjected to future drought screens, possibly longer in duration. 
DP 491 was consistently ranked as the most drought tolerant genotype tested with this method. It is unclear if this ability to survive cyclical drought in the seedling stage will translate into maintained yields under field drought conditions.

Due to the limited fritted clay volumes used in this test, genotypes showing drought tolerance in non-limiting environments due to their ability to access water deep within the soil profile may not have been accurately ranked by this technique. Field evaluations or the use of larger containers may confirm or refute this complication. 


\section{REFERENCES}

Basal, H., P. Bebeli, C. W. Smith, and P. Thaxton. 2003. Root growth parameters of converted race stocks of upland cotton and two BC2F2 Populations. Crop Sci. 43:1983-1988.

Basal, H., C.W. Smith, P.M. Thaxton, and J.K. Hemphill. 2005. Seedling drought tolerance in upland cotton. Crop Sci. 45:766-771.

Cantrell, R.G., C.L. Roberts, and C. Waddell. 2000. Registration of 'Acala 1517-99' Cotton. Crop Sci. 40:1200-1201.

Cook, C.G. and K.M. El-Zik. 1992. Cotton seedling and first-bloom plant characteristics: Relationships with drought-influenced boll abscission and lint yield. Crop Sci. 32:1464-1467.

Heyne, E.G. and A.M. Brunson. 1940. Genetic studies of heat and drought tolerance in maize. J. Amer. Soc. Agron. 32:803-814.

Hosmer, D.W. and S. Lemeshow. 1989. Applied logistic regression. John Wiley \& Sons, New York.

Hunter, J.W., H.H. Laude, and A.M. Brunson. 1936. A method for studying resistance to drought injury in inbred lines of maize. J.Amer. Soc. Agron. 28:694-698.

Johnson, D.A. 1980. Improvement of perennial herbaceous plants for drought-stressed western rangelands. p. 419-433. In N.C. Turner and P.J. Kramer (ed.) Adaptation of plants to water and high temperature stress. John Wiley \& Sons, New York.

Kaul, R. 1966. Effect of water stress on respiration of wheat. Can. J. Bot. 44:623-632.

Kaul, R. 1969. Relations between water status and yield of some wheat varieties. Zeitschrift für Pflanzenzüchtung. 62:145-154.

Kilen, T.C. and R.H. Andrew. 1969. Measurement of drought resistance in corn. Agro. J. 61:669-672.

Lichthardt, J. and T. Weaver. 1985. Tolerance of two and eight-week droughts by range grass seedlings. Proc. Mont. Acad. Sci. 45:46-50.

Liu, S., R. G. Cantrell, J.C. McCarty, Jr., and J. Mcd. Stewart. 2000. Simple sequence repeat-based assessment of genetic diversity in cotton race stock accessions. Crop Sci. 40:1459-1469. 
McCarty, J.C. and J.N. Jenkins. 1993. Registration of 79 day-neutral primitive cotton germplasm lines. Crop Sci. 33:351.

Nepomuceno, A.L., D.M. Oosterhuis, and J.M. Stewart. 1998. Physiological responses of cotton leaves and roots to water deficit induced by polyethylene glycol. Env. Exp. Bot. 40:29-41.

Oosterhuis, D.M. and S.D. Wullschleger. 1987. Osmotic adjustment in cotton (Gossypium hirsutum L.) leaves and roots in response to water stress. Plant Physiol. 84:1154-1157.

Pace, P.F., H.T. Cralle, S.H.M El-Halawany, J.T. Cothren, and S.A. Senseman. 1999. Drought-induced changes in shoot and root growth of young cotton plants. J. Cot. Sci. 3:183-187.

Penna, J.C.V., L.M. Verhalen, M.B. Kirkham, and R.W. McNew. 1998. Screening cotton genotypes for seedling drought tolerance. Gen. Mol. Bio. 21:545-549.

Quisenberry, J.E., W.R. Jordan, B.A. Roark, and D.W. Fryrear. 1981. Exotic cottons as genetic sources for drought resistance. Crop Sci. 21:889-895.

Quisenberry, J.E., B. Roark, and B.L. McMichael. 1982. Use of transpiration decline curves to identify drought-tolerant cotton germplasm. Crop Sci. 22:918-922.

Quisenberry, J.E., C.W. Wendt, J.D. Berlin, and B.L. McMichael. 1985. Potential for using leaf turgidity to select drought tolerance in cotton. Crop Sci. 25:294-299.

Roark, B., J.E. Quisenberry, and J.E. Friesen. 1975. Rate of water loss from detached leaves of cultivars of upland cotton. In Proc. Beltwide Cotton Prod. Res. Conf., New Orleans, LA. 6-8 Jan. 1975. Natl. Cotton Coun., Memphis, TN. (Abstract).

Salter, R., B. Melton, M. Wilson, and C. Currier. 1984. Selection in alfalfa for forage yield with three moisture levels in drought boxes. Crop Sci. 24:345-349.

Sammons, D.J., D.B. Peters, and T. Hymowitz. 1978. Screening soybeans for drought resistance. I. Growth chamber procedure. Crop Sci. 18:1050-1055.

Sammons, D.J., D.B. Peters, and T. Hymowitz. 1979. Screening soybeans for drought resistance. II. Drought box procedure. Crop Sci. 19:719-722.

SAS Institute. 2004. The SAS system for Windows. Release 9.1.3. SAS Inst., Cary, NC.

Tischler, C.R., P.W. Voight, and B.A. Young. 1991. Tray system for measuring drought tolerance of forage grasses. Crop Sci. 31:1696-1699. 
Vasudevan, V. and V. Balasubramaniam. 1964. Germination in osmotic solutions as an index of drought resistance in sorghum. The Madras Agri. J. 52:386-390.

Wright, L.N. 1964. Drouth tolerance - Program-controlled environmental evaluation among range grass genera and species. Crop Sci. 4:472-474.

Wright, L.N., and S.E. Brauen. 1971. Artificial selection for seedling drouth tolerance and association of plant characteristics of Lehmann lovegrass. Crop Sci. 11:324326.

Wright, L.N., and G.L. Jordan. 1970. Artificial selection for seedling drouth tolerance in Boer lovegrass (Eragrostis curvula Nees). Crop Sci. 10:99-102. 


\section{VITA}

Name: $\quad$ Polly Suzanne Longenberger

Address: $\quad$ c/o Dr. C. Wayne Smith

Texas A\&M University

Department of Soil and Crop Sciences

2474 TAMUS

College Station, TX 77843-2474

Education:

2005 M.S., Plant Breeding, Texas A\&M University, College Station, TX.

2003 B.S., Agroecosystems Science, The Pennsylvania State University, University Park, PA.

Publications:

Longenberger, P.S., C.W. Smith, and P. Thaxton. 2005. Development of a screening method for drought tolerance in cotton seedlings. In Proc. Beltwide Cotton Prod. Res. Conf., Natl. Cotton Coun., Memphis, TN. (Abstract). 